\title{
Perception of improvement after orthognathic surgery: the important variables affecting patient satisfaction
}

\author{
Jan Rustemeyer $\cdot$ Ziyad Eke $\cdot$ Andreas Bremerich
}

Published online: 20 March 2010

(C) The Author(s) 2010. This article is published with open access at Springerlink.com

\begin{abstract}
Purpose We evaluated which factors affect patient satisfaction and if patient expectations were fulfilled after orthognathic surgery.

Methods Questionnaires consisting of 14 questions were given 1 year after bimaxillary osteotomy for class-III correction to subjects. Six questions were answered using an 11-point rating scale based on a visual analog scale (VAS; $0=$ poor; $10=$ excellent). Also included were seven closed-form questions with yes/no answers, as well as one open question for 'further remarks'. Sagittal and vertical cephalometric parameters were determined on postoperative cephalograms. Results Seventy-seven patients (37 females, 40 males; mean age, $23.4 \pm 4.9(\mathrm{SD})$ years) responded. The intention to undergo surgery only for aesthetic improvement was noted in $11.9 \%$ of patients; only improvement of chewing function in $15.5 \%$; both in $71.4 \%$; and none/don't know in $2.6 \%$. Postoperative satisfaction was rated (in means) with $8.13 \pm 1.97$ on VAS and correlated significantly with the opinions of friends and relatives. Facial aesthetics was rated $5.6 \pm 1.2$ before surgery and $8.1 \pm 1.5$ after surgery $(p=0.04)$. Preoperative chewing function was rated $5.65 \pm 1.8$ and $8.03 \pm$ 1.51 after surgery $(p=0.014)$. TMJ disorders or hypoesthesia had no negative impacts. Cephalometric analyses revealed a significantly lower $\operatorname{SNB}\left(75.3^{\circ} \pm 2.7^{\circ} ; p=0.033\right)$ in patients rating lower than grade 7 for overall satisfaction. For SNA and ArGoMe, no significant differences were observed. Conclusion The most distinctive factors for patient satisfaction after orthognathic surgery were chewing function and facial
\end{abstract}

J. Rustemeyer $(\bowtie) \cdot$ Z. Eke $\cdot$ A. Bremerich

Department of Oral and Maxillofacial Surgery,

Medical Centre Bremen-Mitte,

Bremen, Germany

e-mail: janrustem@gmx.de aesthetics with respect to the lower face. Function, aesthetics, and even psychological aspects should be considered equally when planning surgery.

Keywords Patient satisfaction - Orthognathic surgery · Bimaxillary osteotomy · Aesthetics · Chewing function . Visual analog scale

\section{Introduction}

Skeletal disfigurement of the face has a negative effect on many aspects of life. These include social interactions, opportunities, choice of profession, choice of partner, and personality characteristics [1,2]. Subjects with malocclusion, particularly those in need of surgical corrections, have a lower healthrelated quality-of-life (QoL) and are more anxious [3-5].

It therefore seems reasonable to offer orthognathic surgery as appropriate treatment to correct a disfigurement if it is subjectively perceived as a handicap, in part to improve the psychology and QoL of the patient. This leads to the consideration that patients' perception of the quality of orthognathic surgery is dependent upon several factors. These are not only aesthetics and function, but also psychological aspects. As a consequence, patients' preoperative expectations of orthognathic surgery and postoperative outcome could offer discrepancies if patients are not clearly informed of what is possible and what is not.

It has been known for decades that postoperative dissatisfaction is not necessarily related to the skill of the surgeon; it results primarily from failure of communication between the surgeon and patient [6]. The values of individual patients have an important role in characterizing the postoperative result, whereas objective parameters of outcome and patient satisfaction may be discordant. A patient-centered approach 
to examining the outcome of medical therapy is an important supplement to the study of morphological and physiological responses to treatment in general because the success of treatment must also be defined in the context of the patient's perceptions of what was achieved [7].

We evaluated which factors have different subjective and objective values with regard to patient satisfaction after bimaxillary osteotomies. We also assessed if patient expectations were fulfilled, as well as their opinions about the result of treatment and side-effects. A questionnaire was designed to assess patient perceptions of problems and gains in oral function, external appearance, and inter-personal relationships before and after surgery. The questionnaire consisted of easyto-understand questions. It was kept concise so as not to overtax patients, and not to cause bias and reduce compliance. It was structured to recall their feelings before and after surgery.

\section{Patients and methods}

Ethical approval of the study protocol

Approval for the study was given by the Ethics Committee of the Medical Association of the state. All participants were informed about the aims and protocol of the study.

Planning and surgery

Simulation of the surgery was done on a pre-surgical cephalogram for each subject using planning software devices (Onxy Ceph Version 2.7.19; Image Instruments, Chemnitz, Germany). Cephalometric prediction was aimed at achieving normal values according to the cephalometric analysis described by Hasund and Jansen [8]. A 'mock operation' was carried out with study models mounted onto an adjustable articulator (SAM III, Sam-dental, Gauting, Germany) for threedimensional planning and manufacturing of the interocclusal positioning wafers (splints). The result of the mock operation was demonstrated and explained to each subject.

All patients in the study underwent bimaxillary osteotomies with advancement of the maxilla by the Le Fort I osteotomy procedure and setback of the mandible by bilateral sagittal split ramus osteotomy for anterior/posterior and vertical skeletal corrections for class-III deficiency. Condylar positioning devices were not used. Patients remained in hospital for 5 days after surgery. Rigid fixation and an interocclusal splint were applied for 2 weeks and afterwards patients wore light training elastics for 2 weeks in total.

The following parameters were determined on postoperative cephalograms, sagittal: SNA (prognathia angle of maxilla; normal, $82^{\circ} \pm 3^{\circ}$ ); SNB (prognathia angle of mandible; normal, $80^{\circ} \pm 3^{\circ}$ ); and vertical: ArGoMe (mandibular jaw angle; normal, $122^{\circ} \pm 2^{\circ}$ ).
Questionnaire

Questionnaires with accompanying letters explaining the importance of information concerning patient perceptions for the improvement of orthognathic surgery were given to patients. Stamp-addressed envelopes were sent to the last known addresses of 110 non-growing adult Caucasian patients. Exclusion criteria were patients with mature cleft lip and palate and craniofacial syndromes, and patients who had undergone orthognathic surgery without orthodontic treatment or with distractor devices.

The questionnaire consisted of 14 questions and was given approximately 1 year after surgery $(13.2$ (mean) \pm 2.1 (standard deviation) months) to eliminate postoperative effect on the patient wellbeing (e.g., intermaxillary fixation, edema or pain) and to ensure continuity in the responses. All patients had their orthodontic appliances removed by the time of survey. In six questions, patient satisfaction; the opinion of relatives on the result of treatment; overall subjective findings regarding function of the temporomandibular joint (TMJ); and masticatory efficiency were rated by patients on an 11-point scale based on a visual analog scale (VAS; $0=$ poor, $10=$ excellent). The questionnaire also included seven closed-form questions with yes/no alternatives related to patient intentions to undergo orthognathic surgery and evaluation of the results; patient self-confidence; and willingness to recommend orthognathic surgery to others. There was one open question for 'further remarks' from patients.

\section{Statistical analyses}

Kolmogorov-Smirnov normality test was performed to determine if the samples conform to normal distribution. Differences between subgroups and correlations between variables were calculated by the chi-square test, $t$ test, and Pearson correlation (SPSS for Windows, version 15.0, SPSS Incorporation, Chicago, IL, USA). Differences were considered significant if $p<0.05$ and highly significant if $p<0.001$.

\section{Results}

Questionnaire

Seventy-seven patients (37 females and 40 males; mean age, $23.4 \pm 4.9$ (SD) years; range, 17-34 years) completed and returned questionnaires during 5 weeks (response rate, $70 \%$ ). All 77 questionnaires were ready for evaluation in this study.

Overall, no significant differences were noted between females and males or for different ages for any answer. The 
questionnaire with the resulting response for each question is given below.

Q 1: What was your primary intention to undergo orthodontic surgery? Please mark one of the four given answers:

Aesthetic improvement only-improvement of chewing function only-both-none/don't know

The answer "aesthetic improvement only" was marked in $11.9 \%$ of subjects; "improvement of chewing function only" in $15.5 \%$; "both" in $71.4 \%$; and "none/don't know" in $2.6 \%$.

Q 2: How do you evaluate your postoperative result in total? Please mark one of the four given answers:

Aesthetic improvement only-improvement of chewing function only-both-none/don't know

The answer "aesthetic improvement only" was marked in $15.6 \%$ of subjects; "improvement of chewing function only" in $5.1 \%$; "both" in $75.4 \%$; and "none/don't know" in $4 \%$. There was a significant difference for "improvement of chewing function only" between Q1 and this question ( $p=$ 0.022 ) due to a shift from $n=8$ patients towards the category "both". A shift from the category "both" to other categories $(n=4)$ was not significant.

Q 3: How do you feel exactly about the surgical outcome of your operation? Please mark one grade of the scale from 0 (poor) to 10 (excellent).

$0-1-2-3-4-5-6-7-8-9-10$

The mean rating was $8.13 \pm 1.97 ; 12.9 \%$ of subjects marked the surgery outcome as grade 10 ("excellent").

Q 4: How do your relatives and friends feel in total about the surgical outcome of your operation? Please mark one grade of the scale from 0 (poor) to 10 (excellent).

\section{$0-1-2-3-4-5-6-7-8-9-10$}

The mean rating was $8.48 \pm 1.09$. The higher mean rating compared with the answer to Q3 was not significant. Significant differences to Q3 occurred within grade 8 ( $p=$ $0.034)$ and grade $10(28.6 \%$; $p=0.033$; Fig. 1$)$. Rating in Q3 and in this question correlated significantly with each other (Pearson correlation $\left.\left(P_{\text {corr }}\right)=0.867 ; p=0.001\right)$.

Q 5: How would you assess your facial esthetics before surgery? Please mark one grade of the scale from 0 (poor) to 10 (excellent).

$0-1-2-3-4-5-6-7-8-9-10$

The mean rating was $5.65 \pm 1.8$.

Q 6: How would you assess your facial esthetics after surgery? Please mark one grade of the scale from 0 (poor) to 10 (excellent).

$0-1-2-3-4-5-6-7-8-9-10$

The mean rating was $8.03 \pm 1.51$. This value was significantly higher than the value given in Q5 $(p=0.014)$. In particular, the ratings for grade 8 were significantly higher $(p=0.014)$, and ratings for grades 9 and 10 were more highly significantly compared with Q5 ( $p<0.001$; Fig. 2).
Q 7: Do you have more self-confidence since surgery? (Yes or No).

A total of $67.5 \%$ of subjects answered "Yes".

Q 8: Assuming that orthognathic surgery is indicated, would you recommend it to others? (Yes or no.)

A total of $73 \%$ of subjects answered "Yes".

Q 9: How would you assess your chewing function before surgery? Please mark one grade of the scale from 0 (poor) to 10 (excellent).

$0-1-2-3-4-5-6-7-8-9-10$

The mean rating was $5.57 \pm 1.24$ (SD). No correlation with Q5 was observed $\left(P_{\text {corr }}=0.503 ; p=0.114\right)$.

Q 10: How would you assess your chewing function today? Please mark one grade of the scale from 0 (poor) to 10 (excellent).

$0-1-2-3-4-5-6-7-8-9-10$

The mean rating was $8.23 \pm 1.56$. This value was significantly higher than the value given in Q9 $(p=0.015)$. In particular, the ratings for grades 9 and 10 were more highly significantly in this question compared with Q9 $(p<0.001 ;$ Fig. 3$)$. In addition, a highly significant correlation could be found with Q6 $\left(P_{\text {corr }}=0.947 ; p<0.001\right)$.

Q 11: Do you have "numbness" or "prickle-sensations" in your face, lower or upper lip area or gums today? (Yes or no.)

A total of $54.6 \%$ of subjects answered "Yes". The mean rating of these patients in Q3 was 7.76 \pm 0.91 . Overall, there was no significant difference to patients who answered "No" ( $8.57 \pm 1.43 ; p=0.562)$. Only rating counts for grade 9 presented a significant difference between both groups with a higher count of patients who answered "No" $(p=0.002)$.

Q 12: Did you have any pain in the temporomandibular joint area or any restrictions in mouth opening before surgery? (Yes or no.)

A total of $20.8 \%$ of subjects answered "Yes".

Q 13: Do you have now any pain in the temporomandibular joint area or any restrictions in mouth opening today? (Yes or no.)

'A total of $18.2 \%$ of subjects answered "Yes"; $75 \%$ of patients who answered "Yes" in Q12 answered "No" and $16.4 \%$ of patients who answered "No" in Q12 answered "yes" in this question. The mean rating of these patients in Q3 was $7.57 \pm 1.18$. In total, there was no significant difference to patients who answered "No" $(8.25 \pm 1.12$; $p=0.995$ ). Only rating for grade 8 presented a significant difference between both groups with a higher count of patients who answered "No" $(p=0.028)$.

O 14: Do you have further remarks or annotations concerning the orthognathic surgery service?

A total of $75.3 \%(n=58)$ of subjects had no further remarks or annotations; $24.7 \%(n=19)$ criticized various non-medical aspects such as dysfunction of internet access, phone or television, flavor of the diet, and nursery care in general. 
Fig. 1 Perceptions of overall postoperative outcomes of patients and friends/relatives on a visual analog scale $(V A S)$. *Significant at $p<0.05$ for each category

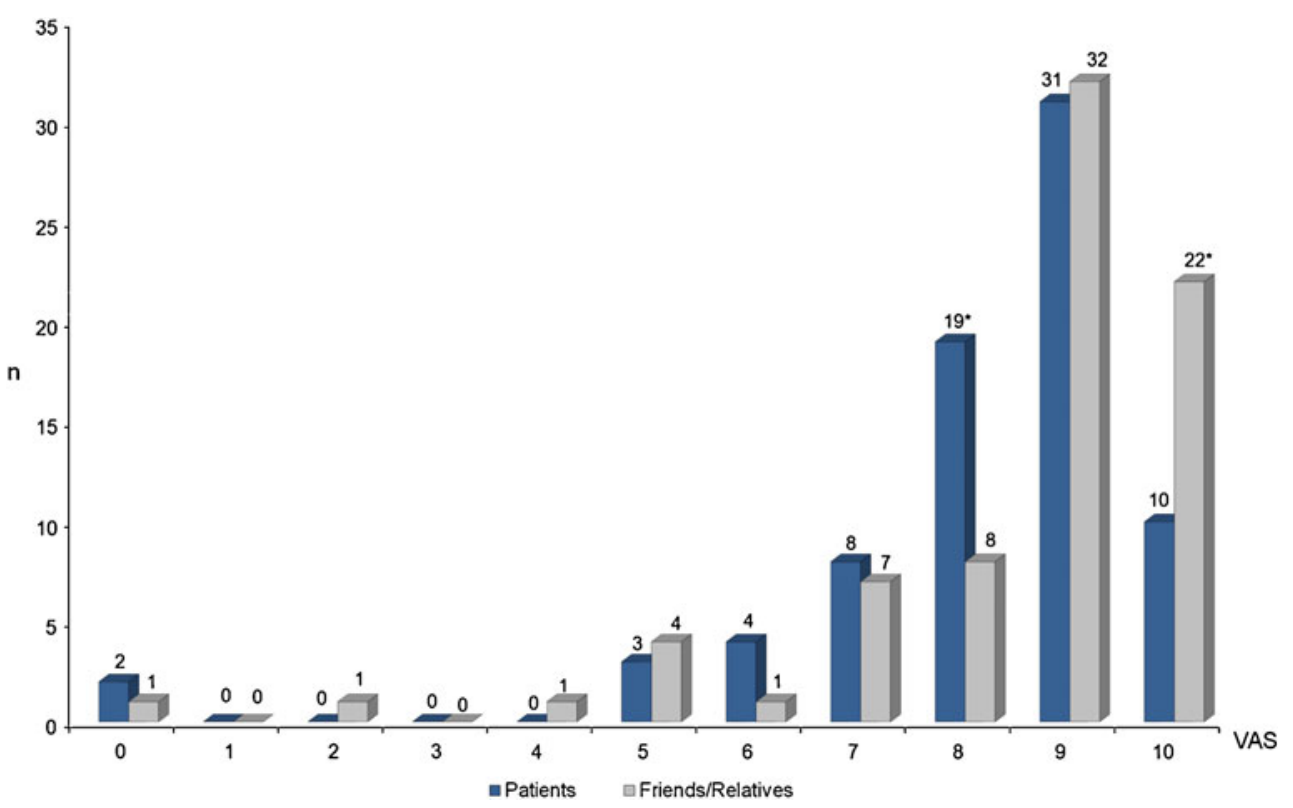

Cephalometric outcomes and patient satisfaction

Postoperative cephalometric analyses revealed a significantly lower $\operatorname{SNB}\left(75.3^{\circ} \pm 2.7^{\circ} ; p=0.033\right)$ in patients rating lower than grade 7 compared with patients rating 7 or higher in Q3 $\left(81.2^{\circ} \pm 2.58^{\circ}\right.$; Fig. 4). No significant difference was obtained between the SNB in patients rating lower than grade 7 and the normal range of SNB ( $p=$ 0.698). With respect to SNA and ArGoMe, no significant differences occurred between the ratings of the patients.

\section{Discussion}

Dentofacial deformity affects patient QoL in the community. In particular, dentoskeletal class-III malocclusion results in unaesthetic alterations of soft tissues which may cause psychological and inter-personal problems [9].
Our results revealed that satisfaction after orthognathic surgery in former class-III patients was, in general, high. A total of $77.9 \%$ of all patients rated postoperative outcomes with grades 8,9 , and 10 on the VAS 1 year after surgery. With respect to recommending surgery, $73 \%$ considered advising other patients to undergo orthognathic surgery. The high satisfaction rate was in accordance with that reported in previous studies, which ranged between $70 \%$ [10] and $87 \%$ [11], and which was also higher than that reported among pre-treatment and no-treatment control groups [4]. Hence, positive changes occurred in the personality profiles of patients. There was an obvious improvement in self-confidence in $67.5 \%$ of patients as a result of an improved appearance and an improved chewing function. Patients responded that surgical treatment had had a great effect on family and friends. Satisfaction of those correlated significantly with patients' satisfaction. From the present study, we understand that patients' mental attitude

Fig. 2 Comparison of the preand postoperative perceptions of aesthetics in patients using the VAS. ${ }^{*}$ Significant at $p<0.05$ for each category. ${ }^{* *}$ Highly significant at $p<0.001$ for each category

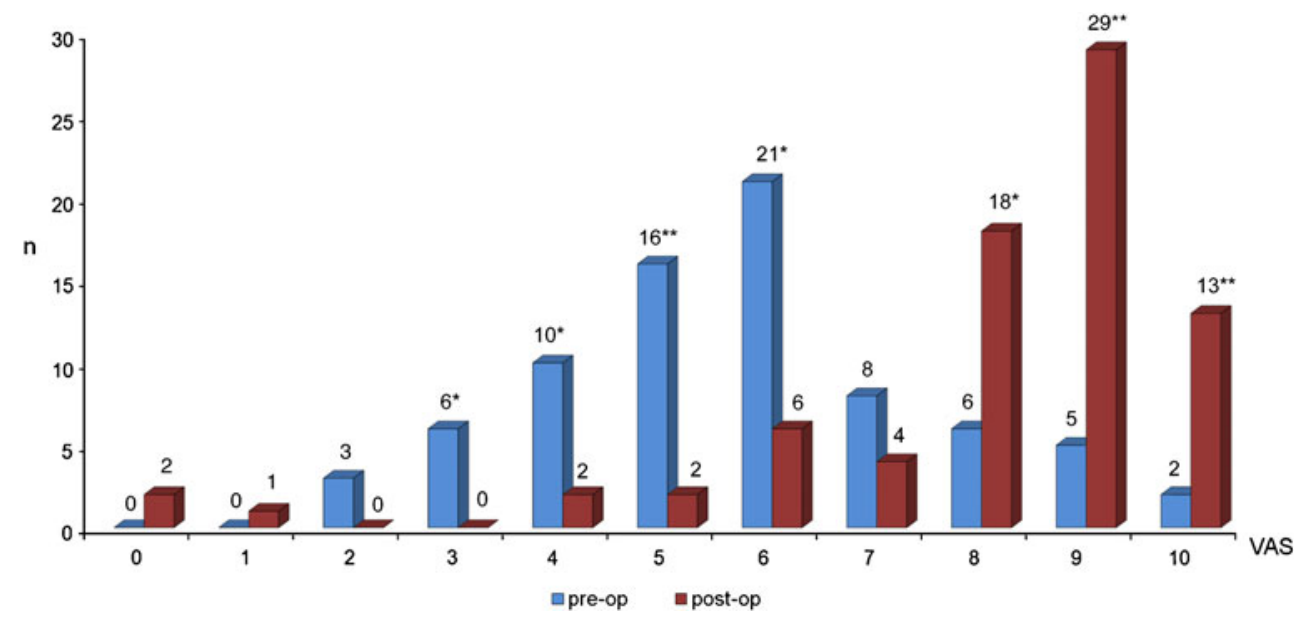


Fig. 3 Comparison of the preand postoperative perceptions of chewing function in patients using the VAS. *Significant at $p<0.05$ for each category.

$* *$ Highly significant at $p<0.001$ for each category

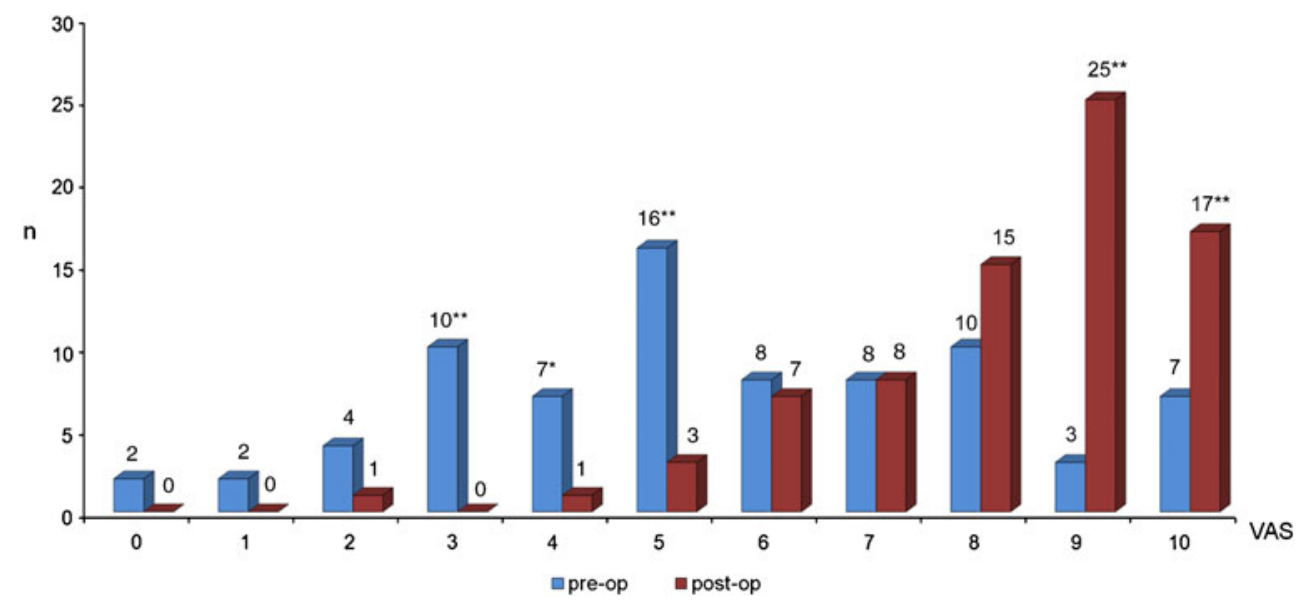

about his/her postoperative appearance may be influenced by the support network after surgery. In contrast, the prevalence of postoperative problems such as numbness, prickle-sensations, pain in the TMJ area, or restrictions in mouth-opening had no overall effect on satisfaction, which remained high. However, a negative tendency on patient ratings could be observed if postoperative problems were raised and persisted. These results were in accordance with the findings of other studies [11-13]; even if sensory disturbance was frequently observed, most patients (particularly in the younger age groups) seemed to adapt. The primary determinant of satisfaction with surgery was whether or not the outcome was perceived to be an aesthetic improvement. If there was an aesthetic improvement in facial features, the satisfaction was high regardless of functional problems.

One result of this survey was that a correlation between age, sex, and response to the questionnaire was not observed. When these findings were compared with other studies including patients with different dysgnathic classes, it was apparent that age and sex had a significant influence on the expectations and surgical outcome among populations. More young males than females wanted functional improvement, whereas younger females than males hoped for improvement in self-confidence [14]. Older female patients displayed improved self-esteem and diminished depressive symptoms due to surgical intervention; whereas older male patients showed no alteration in self-esteem or depression with surgical intervention [15]. This discrepancy with other studies may be because in this study the population consisted of patients aged primarily around 23 years, so elderly subjects were not adequately represented, and perhaps no obvious significant difference between females and males exists in class-III patients. These aspects had no influence on the significance of the present study because the motives and concerns of patients that initiated treatment were improvement of aesthetics and chewing function (71.4\%) and results were in accordance with findings in recent studies [16, 17]. However, both intentions may not be natural but instead be dependent upon regional distinctions. For example, in an Indian population, it was found that the primary motivation of many patients who seek orthognathic surgery was aesthetics and not for correction of functional disability [18]. In addition, problems related to the TMJ may be the reason for undergoing treatment in only a minority of subjects [13], even though $20.8 \%$ of all patients presented signs of disorder of the TMJ in the present study. Surgical correction of dentofacial deformities has been shown to relieve the signs and symptoms relating to TMJ dysfunction such as pain or restriction of mouth-opening in $75 \%$ of patients. Nevertheless, a small number of patients continued to experience TMJ problems and $16.4 \%$ even developed new symptoms of TMJ disorders after surgery. In comparison, other studies reported similar data demonstrating $16-20 \%$ of orthognathic surgery patients with TMJ symptoms before surgery. However, a wide range from $40 \%$ to $75 \%$ of these symptomatic patients reported fewer or no symptoms after surgery, and about $12 \%$ of preoperatively asymptomatic patients also developed TMJ symptoms after surgery [19, 20]. In comparison, patients with asymmetrical deformities showed significant more TMJ symptoms after surgery leading to less satisfaction 1 year postoperatively [21].

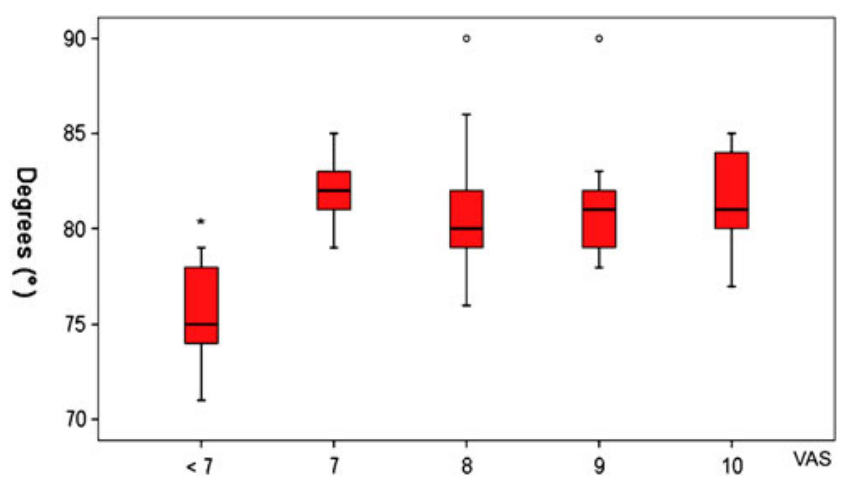

Fig. 4 Postoperative cephalometric analyses of SNB and patient perceptions of overall outcome on VAS. empty circle Indicates outliers. *Significant at $p<0.05$ 
Patients in this study who reported TMJ impairment were offered complementary treatment such as physiotherapy and splint therapy.

It is well-known that satisfaction and the perception of surgical outcome are dependent upon preoperative expectations and the degree to which the procedure is explained by the operating staff $[22,23]$. Patients who are psychologically distressed before orthognathic surgery tend to report a higher overall recovery burden and, on average, experience more difficulty with symptoms, social/selfconcerns, and general health in the first 1-2 months after surgery [24]. However, various factors may modulate postoperative satisfaction in a positive direction. Written information about possible sequelae and the recovery period, and patient consent to the surgical procedure may be helpful and may reduce postoperative dissatisfaction [14, 25]. In surveys carried out by Türker et al. [10] and Williams et al. [16], subjects admitted for orthognathic surgery talked to patients who had previously undergone surgery and, as a result, they felt more ready for surgery and expressed great satisfaction with the outcome. During conservations, the previously operated participants quoted their experiences, which had a contributory effect on the emotional preparation of the patients about to undergo surgery. This activity did not create negative impressions in the patients because it did not cause distress or anxiety. Arranging for new patients to talk to patients who have undergone treatment may be difficult to organize as part of a busy joint clinic and also raises issues of patient confidentiality. In addition, viewing treatment simulation before surgery did not, on average, negatively affect perception of symptoms or satisfaction after surgery [24]. A high level of patient satisfaction (up to 89\%) may also be achieved with simultaneous procedures, including orthognathic surgery, septoplasty, removal of third molars, and even liposuction [26]. However, "All in one" procedures as a mean to improve patient satisfaction do not fit with our philosophy of orthognathic surgery. We assume, besides an increased prevalence of morbidity, results may be harder to predict and reciprocally, satisfaction may decrease. Nevertheless, two of 42 patients (5\%) undergoing simultaneous procedures in the study of Posnick and Wallace [26] were dissatisfied despite the absence of surgical or orthodontic complications, and despite the clinicians' feelings that the results were an improvement. These findings are comparable with the data of Türker et al. [10] showing 3\% of unsatisfied patients, and with our data demonstrating two patients (2.6\%) who were unsatisfied and rated with grade 0 (poor) for the overall surgical outcome. Interestingly, there were no patients in the present study who rated on the scale for overall satisfaction grades $1-4$, so both patients rating 0 represented obviously extreme views. If the response rates of surveys including questionnaires are low, responders may not be representative of non-responders. Furthermore, responders may represent individuals who have extreme views. Although a significant proportion of patients were lost to follow-up, a response rate of $70 \%$ was achieved in the present study. This could be regarded as typical for this type of study $[27,28]$. Considering the mobility of this group of patients (who tend to undergo surgery at school-leaving age), this should be regarded as a good response rate for this population.

After orthognathic surgery, most patients report an improvement in self-confidence, body and facial image, and social adjustment, but some patients are only temporarily satisfied and become litigious or violent [2]. Patients who receive inadequate explanation of the surgery are prone to be emotionally unprepared and anxious after surgery, but orthognathic surgery may not be beneficial for patients who assume that it will solve most of their problems. The latter group may suffer from body dysmorphic disorder (BDD) [29]. Because of the delusional content of the disorder, patients with BDD lack insight in the unreal nature of their bodily concerns. They are often not (or only temporarily) satisfied with the result of surgery. In maxillofacial surgery, even $10 \%$ of patients could be screened positive for BDD. The high prevalence of problems related to psychosocial functioning and the high occurrence of BDD in maxillofacial patients makes a referral of these patients to psychiatrists necessary for appropriate treatment. This minimizes their opportunities for several referrals, litigation, and unnecessary orthognathic surgery.

The assessment of facial beauty is subjective, but the assessment of facial proportions should be undertaken objectively. Disproportionate human faces are unattractive, whereas proportionate features are acceptable, even if not always attractive [30]. Treatment, if based on cephalometric evaluations alone, can result in inadequate correction of facial aesthetics and patient dissatisfaction. Soft-tissue changes after skeletal advancement or setback should be addressed and cephalometric indications compared with aesthetic clinical indications and (if possible) skeletal planning must be corrected by the aesthetic needs so that aesthetic and functional success can be reached simultaneously [31, 32]. We previously reported that in cases of bimaxillary osteotomies, differences between predicted and postoperative results could be measured with a range of $1-2^{\circ}$ for SNA, $\mathrm{SNB}$, and ArGoMe [33]. This small difference is not apparent for facial aesthetics. However, it is highly interesting to find that in the present survey, patients who rated significantly lower on the VAS $(<7)$ were more dissatisfied when presenting a lower SNB in the postoperative cephalogram compared with patients who rated grade 7 or higher. This should be seen only as a tendency because the observed significantly lower SNB in these patients covered the lower normal range and was in accordance with predictions. For 
SNA and ArGoMe, similar relationships were not detectable. One reason may be (as supported by the literature) that up to $30 \%$ of patients had difficulty getting used to their appearance even 24 months after surgery [10]. In particular, correction of the lower facial area with a mandible setback in class-III patients for an improved facial profile with a reduced labiomental angle and accentuation of the chin could lead to an extreme alteration of appearance, more than movement of the maxilla alone could provide [31]. These alterations rely more on the reduction of the sagittal SNB angle as on the vertical ArGoMe angle. In class-III cases in which SNB is surgically narrowed to the lower normal range, profile changes are very radical and may contribute significantly to the delay in getting used to the new appearance. Therefore, it may result in more dissatisfaction 1 year after surgery. Additionally, the opinions of friends and relatives affect the satisfaction of the patient, and friends and relatives may also have problems getting used to the new appearance of the patient.

\section{Conclusion}

The questionnaire designed for this survey showed results which were comparable with studies using fewer [13] or more questions $[11,16,18]$ for a smaller $[10,15,21,28]$ or larger population $[4,13]$. It was therefore suitable to assess patient satisfaction after routine orthognathic surgery. Most patients who underwent orthognathic surgery readily accepted the change in their appearance and were highly satisfied with the outcome. A SNB in the lower normal range leads to a lower satisfaction in patients. This result has to be validated in further studies. However, it emphasizes the aesthetic and psychological factors involved in planning orthognathic surgery.

Conflict of interest The authors declare they have no conflict of interest.

Open Access This article is distributed under the terms of the Creative Commons Attribution Noncommercial License which permits any noncommercial use, distribution, and reproduction in any medium, provided the original author(s) and source are credited.

\section{References}

1. Macgregor FC (1990) Facial disfigurement: problems and management of social interaction and implications for mental health. Aesthet Plast Surg 14:249-257

2. Barbosa AL, Marcantonio E, Barbosa CE, Gabrielli MF, Gabrielli MA (1993) Psychological evaluation of patients scheduled for orthognathic surgery. J Nihon Univ Sch Dent 35:1-9
3. Ozgür F, Tuncali D, Güler Gürsu K (1998) Life satisfaction, selfesteem, and body image: a psychosocial evaluation of aesthetic and reconstructive surgery candidates. Aesthet Plast Surg 22:412-419

4. Lazaridou-Terzoudi T, Kiyak HA, Moore R, Athanasiou AE, Melsen B (2003) Long-term assessment of psychologic outcomes of orthognathic surgery. J Oral Maxillofac Surg 61:545-552

5. Azuma S, Kohzuki M, Saeki S, Tajima M, Igarashi K, Sugawara J (2008) Beneficial effects of orthodontic treatment on quality of life in patients with malocclusion. Tohoku J Exp Med 214:39-50

6. Peterson LJ, Topazian RG (1974) The preoperative interview and psychological evaluation of the orthognathic surgery patient. J Oral Surg 32:583-588

7. Phillips C (1999) Patient-centered outcomes in surgical and orthodontic treatment. Semin Orthod 5:223-230

8. Hasund A, Jansen I (1978) Der kieferorthopädische Behandlungsplan. Organisation und Dokumentation (Orthodontic treatment planning. Organization and documentation). Hanser, München

9. Cunningham SJ, Garratt AM, Hunt NP (2000) Development of a co ndition-specific quality of life measure for patients with dentofacial deformity: I. Reliability of the instrument. Community Dent Oral Epidemiol 28:195-201

10. Türker N, Varol A, Ogel K, Basa S (2008) Perceptions of preoperative expectations and postoperative outcomes from orthognathic surgery: part I: Turkish female patients. Int J Oral Maxillofac Surg 37:710-715

11. De Clercq CA, Neyt LF, Mommaerts MY, Abeloos JS (1998) Orthognathic surgery: patients' subjective findings with focus on the temporomandibular joint. J Cranio-maxillo-facial Surg 26:29-34

12. Kiyak HA, Hohl T, West RA, McNeill W (1984) Psychological changes in orthognathic surgery patients: a 24 month follow-up. J Oral Maxillofac Surg 42:506-512

13. Espeland L, Høgevold HE, Stenvik A (2008) A 3-year patientcentred follow-up of 516 consecutively treated orthognathic surgery patients. Eur J Orthod 30:24-30

14. Siow KK, Ong ST, Lian CB, Ngeow WC (2002) Satisfaction of orthognathic surgical patients in a Malaysian population. J Oral Sci 44:165-171

15. Nicodemo D, Pereira MD, Ferreira LM (2008) Self-esteem and depression in patients presenting angle class III malocclusion submitted for orthognathic surgery. Med Oral Patol Oral Cir Bucal 13:E48-E51

16. Williams AC, Shah H, Sandy JR, Travess HC (2005) Patients' motivations for treatment and their experiences of orthodontic preparation for orthognathic surgery. J Orthod 32:191-202

17. Modig M, Andersson L, Wårdh I (2006) Patients' perception of improvement after orthognathic surgery: pilot study. Br J Oral Maxillofac Surg 44:24-27

18. Narayanan V, Guhan S, Sreekumar K, Ramadorai A (2008) Selfassessment of facial form oral function and psychosocial function before and after orthognathic surgery: a retrospective study. Indian J Dent Res 19:12-16

19. Kerstens HC, Tuinzing DB, van der Knast WA (1989) Temporomandibular joint symptoms in orthognathic surgery. J Craniomaxillo-facial Surg 17:215-218

20. Pahkala RH, Kellokoski JK (2007) Surgical-orthodontic treatment and patients' functional and psychosocial well-being. Am J Orthod Dentofacial Orthop 132:158-164

21. Al-Ahmad HT, Al-Omari IK, Eldurini LN, Suleiman AA (2008) Factors affecting satisfaction of patients after orthognathic surgery at a University Hospital. Saudi Med J 29:998-1003

22. Jensen SH (1978) The psychosocial dimensions of oral and maxillofacial surgery: a critical review of the literature. J Oral Surg 36:447-453

23. Kiyak HA, NcNeill RW, West RA (1985) Emotional impact of orthognathic surgery and conventional orthodontics. Am J Orthod 88:224-234 
24. Phillips C, Kiyak HA, Bloomquist D, Turvey TA (2004) Perceptions of recovery and satisfaction in the short term after orthognathic surgery. J Oral Maxillofac Surg 62:535-544

25. Kim S, Shin SW, Han I, Joe SH, Kim MR, Kwon JJ (2009) Clinical review of factors leading to perioperative dissatisfaction related to orthognathic surgery. J Oral Maxillofac Surg 67:2217-2221

26. Posnick JC, Wallace J (2008) Complex orthognathic surgery: assessment of patient satisfaction. J Oral Maxillofac Surg 66:934-942

27. Asch DA, Kathryn Jedrziewski M, Christakis NA (1997) Response rates in mail surveys published in medical journals. J Clin Epidemiol 50:1129-1136

28. Kharrat K, Assante M, Chossegros C, Cheynet F, Blanc JL, Guyot L, Richard O (2006) Patient perception of functional and cosmetic outcome of orthognathic surgery. Retrospective analysis of 45 patients. Rev Stomatol Chir Maxillofac 107:9-14

29. Vulink NC, Rosenberg A, Plooij JM, Koole R, Bergé SJ, Denys D (2008) Body dysmorphic disorder screening in maxillofacial outpatients presenting for orthognathic surgery. Int $\mathrm{J}$ Oral Maxillofac Surg 37:985-991

30. Naini FB, Cobourne MT, McDonald F, Donaldson AN (2008) The influence of craniofacial to standing height proportion on perceived attractiveness. Int J Oral Maxillofac Surg 37:877885

31. Marșan G, Cura N, Emekli U (2009) Soft and hard tissue changes after bimaxillary surgery in Turkish female Class III patients. J Cranio-maxillo-facial Surg 37:8-17

32. Becelli R, Renzi G, Carboni A, Cerulli G, Perugini M (2002) Evaluation of the esthetic results of a 40-patient group treated surgically for dentoskeletal class III malocclusion. Int J Adult Orthodon Orthognath Surg 17:171-179

33. Rustemeyer J, Groddeck A, Zwerger S, Bremerch A (2009) The accuracy of two-dimensional planning for routine orthognathic surgery. Br J Oral Maxillofac Surg. doi:10.1016/j.bjoms. 2009.06.018 